\title{
Characteristics of the Aging Skin
}

\author{
Miranda A. Farage, ${ }^{1, *}$ Kenneth W. Miller, ${ }^{1}$ Peter Elsner, ${ }^{2}$ \\ and Howard I. Maibach ${ }^{3}$ \\ ${ }^{1}$ The Procter and Gamble Company, Cincinnati, Ohio \\ ${ }^{2}$ Klinik Fur Dermatologic, Jena, Germany. \\ ${ }^{3}$ Department of Dermatology, University of California, San Francisco, California.
}

Significance: Although most researches into the changes in skin with age focus on the unwelcome aesthetic aspects of the aging skin, skin deterioration with age is more than a merely cosmetic problem. Although mortality from skin disease is primarily restricted to melanoma, dermatological disorders are ubiquitous in older people with a significant impact on quality of life. The structural and functional deterioration of the skin that occurs with age has numerous clinical presentations, ranging from benign but potentially excruciating disorders like pruritus to the more threatening carcinomas and melanomas.

Recent Advances: The degenerative changes that occur in the aging skin are increasingly understood at both the molecular and cellular level, facilitating a deeper understanding of the structural and functional deterioration that these changes produce.

Critical Issues: A loss of both function and structural stability in skin proceeds unavoidably as individuals age, which is the result of both intrinsic and extrinsic processes, which contribute simultaneously to a progressive loss of skin integrity. Intrinsic aging proceeds at a genetically determined pace, primarily caused by the buildup of damaging products of cellular metabolism as well as an increasing biological aging of the cells. Estrogen levels strongly influence skin integrity in women as well; falling levels in midlife, therefore, produce premature aging as compared with similarly aged men. Extrinsic insults from the environment add to the dermatological signs of aging.

Future Directions: A deeper understanding of the physiological basis of skin aging will facilitate progress in the treatment of the unwelcome sequelae of aging skin, both cosmetic and pathogenic.

\section{SCOPE}

LIFE EXPECTANCY in the United States and other industrialized countries continues to increase and is expected to reach 100 years by about 2025. ${ }^{1}$ Women have longer average life expectancies than do men and can thus soon expect to spend more than one-third of their lifetimes in menopause. ${ }^{2}$ The skin is incredibly durable, but like all other systems, it eventually succumbs to the inexorable effects of aging. The skin is also the most visible indicator of age.

The skin, a full one-sixth of body weight, is a sophisticated and dynamic organ, which serves as a bulwark between the sensitive internal tissues of the body and the external environment. Not a mere barrier, the integument is involved in the maintenance of body temperature and internal hydration, sensory functions, and immunological surveillance.

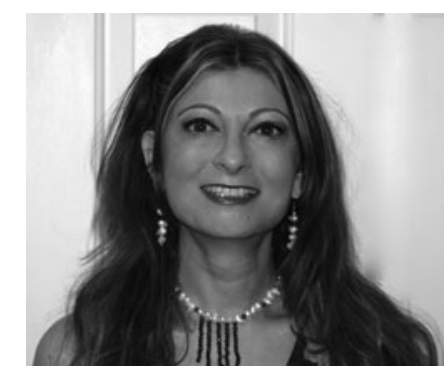

Miranda A. Farage, MSc, PhD

Submitted for publication February 22, 2012. *Correspondence: The Procter and Gamble Company, Winton Hill Business Center, 6110 Center Hill Road, Box 136, Cincinnati, OH 45224 (e-mail: farage.m@pg.com).

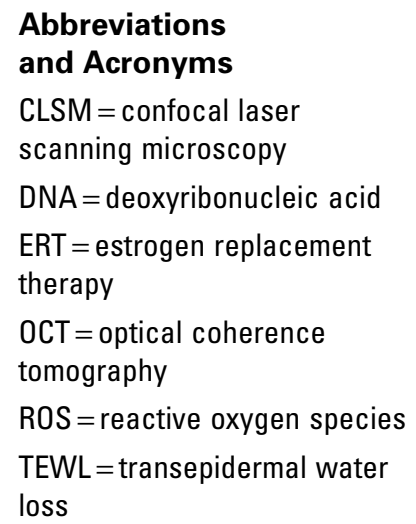


The process of aging affects the skin in multiple ways. The epidermis thins and turnover rate slows dramatically. ${ }^{3}$ Despite a substantial loss of function in aging skin, most skin-aging therapies focus on ending or reversing the unwelcome visible signs of aging. As the population ages, the care of aging skin must shift focus from aesthetic concerns to how the loss of structure and function affects quality of life.

\section{TARGET ARTICLES}

1. Farage MA, Miller KW, Elsner P, and Maibach HI: Functional and physiological characteristics of the aging skin. Aging Clin Exp Res 2008; 20: 195 (Review).

2. Krueger N, Luebberding S, Oltmer M, Streker M, and Kerscher M: Age-related changes in skin mechanical properties: a quantitative evaluation of 120 female subjects. Skin Res Technol 2011; 17: 141.

\section{TRANSLATIONAL RELEVANCE}

Skin ages in both men and women through parallel internal and external processes, which contribute simultaneously to a progressive loss of skin integrity. Structural stability as well as physiological function are affected. Estrogen levels also strongly influence skin integrity in women; falling levels in midlife contribute to earlier signs of aging. ${ }^{8}$ Intrinsic aging proceeds at different rates in all organisms at a genetically determined pace, caused primarily by the buildup of reactive oxygen species (ROS) as a by-product of cellular metabolism. ROS, in turn, cause damage to critical cellular components like membranes, enzymes, and deoxyribonucleic acid (DNA). In addition, as individual ages, skin cells are also biologically aging. Proliferation rates consequently begin to drop in the epidermis, inducing a steady deterioration of skin structure and function. ${ }^{4}$

Exogenous insults also contribute to the aging process. Ultraviolet light, for example, can cause thousands of cellular DNA alterations each day, ${ }^{5}$ causing cumulative damage to skin that amplifies normal chronological decline. Environmental insults in the form of pollution or cigarette smoke also accelerate natural aging processes. ${ }^{6}$

The effects of skin aging, both internal and external, have the potential to produce significant morbidity. Most people over 65, in fact, have at least one skin disorder, and many have two or more. ${ }^{7}$ In addition, the aging of the very visible integument is often psychologically distressing.
Although rarely fatal, dermatological disorders can contribute significantly to a loss of quality of life in an individual's latter years and deserve attention. Understanding the basic physiological processes of aging as well as the spectrum of its effects on the skin will facilitate the efficacy of future treatments.

\section{CLINICAL RELEVANCE}

Aged skin undergoes progressive structural and functional degeneration that leaves it prone to a wide variety of bothersome and possibly even fatal conditions and diseases, including eczema, asteatotic eczema, contact and allergic dermatitis, seborrheic dermatitis, autoimmune diseases with cutaneous manifestations, seborrheic keratoses, and various forms of neoplasms, such as basal and squamous cell carcinoma and malignant melanoma..$^{8-11}$

The majority of senior adults have at least one treatable skin complaint, and many have more. These can be severe and may significantly impact quality of life in the older patient. ${ }^{7,12}$ As the percentage of elderly in the population continues to increase, their dermatological problems increase in clinical significance.

\section{EXPERIMENTAL MODEL OR MATERIAL: ADVANTAGES AND LIMITATIONS}

The human skin is a large and surprisingly complex organ, which not only provides protection for internal tissues, but also facilitates neurosensory, circulatory, and immunological functions at the body surface. As such, evaluation of the structural and functional changes that occur as the body ages relies on a wide variety of direct and indirect measurements of specific skin parameters. Such parameters include biochemical evaluations of skin condition; structural assessments of skin, including thickness, collagen content and turnover, and cell size; as well as functional assessments, such as elasticity, torsion extensibility, neuroperception, transepidermal water loss (TEWL), and proliferation rate. These assessments are achieved through a variety of laboratory and in vivo analyses as well as scanning electron microscopy, confocal laser scanning microscopy (CLSM), optical coherence tomography (OCT), and ultrasound echogenicity. ${ }^{13}$ These methods applied to a human skin model provide the most complete and rigorous understanding of the effects of aging in human beings, and as such is far preferable to using other models. Using human skin as a model, 
however, obviously comes with many constraints that limit the scope of research as well as what specific information can be obtained, as obtaining volunteers and Ethics Board approval adds both time and expense to every study undertaken. Maximizing the study of aging in the human skin while taking advantage of the substantial body of research using other models can contribute to a comprehensive understanding of the underlying physiological processes that result in the age-related deterioration of skin integrity. ${ }^{13}$

\section{DISCUSSION OF FINDINGS AND RELEVANT LITERATURE}

Physiological changes in aged skin include structural and biochemical changes as well as changes in neurosensory perception, permeability, response to injury, repair capacity, and increased incidence of some skin diseases. Although the number of cell layers remains stable ${ }^{14}$ the skin thins progressively over adult life at an accelerating rate. ${ }^{15}$ The epidermis decreases in thickness, ${ }^{16}$ particularly in women and particularly on the face, neck, upper part of the chest, and the extensor surface of the hands and forearms. ${ }^{17}$ Thickness decreases about $6.4 \%$ per decade on average, with an associated reduction in epidermal cell numbers. ${ }^{15,18}$

Keratinocytes, as skin ages, change shape, becoming shorter and fatter, while corneocytes be- come bigger as a result of decreased epidermal turnover. ${ }^{19}$ Enzymatically active melanocytes decrease at a rate of $8 \%$ to $20 \%$ per decade, resulting in uneven pigmentation in elderly skin. ${ }^{20}$ Although the number of sweat glands does not change, sebum production decreases as much as $60 \% .^{13}$

A reduction of the natural water and fat emulsion on the skin is observed, ${ }^{21}$ as is water content in the stratum corneum. ${ }^{16,22,23}$ Global lipid content of the aged skin is reduced as much as $65 \% .{ }^{19}$ Changes in the amino acid composition in aged skin ${ }^{23}$ may reduce the amount of cutaneous natural moisturizing factor, thereby decreasing its capacity for water binding. ${ }^{22}$ Baseline TEWL decreases with age ${ }^{16,24}$ an observation that is believed to be due to the reduction of the water content of aged skin. Recovery of baseline TEWL values after occlusion ${ }^{16}$ or tape stripping ${ }^{25}$ is also slower in older skin, revealing a profound change in barrier integrity despite the fact that barrier function in aged skin under normal conditions appears normal. ${ }^{13}$

The most consistent structural change in aged skin is a flattening of the dermo-epidermal junction by more than a third (Figure 1), ${ }^{26-28}$ which occurs as a result of the loss of dermal papillae ${ }^{27}$ as well as a reduced interdigitation between layers. ${ }^{14}$ This flattening, observable by scanning electron microscopy beginning in the sixth decade, ${ }^{15}$ results in less resistance to shearing forces and an increased vulnerability to insult. ${ }^{14}$ The smaller contiguous

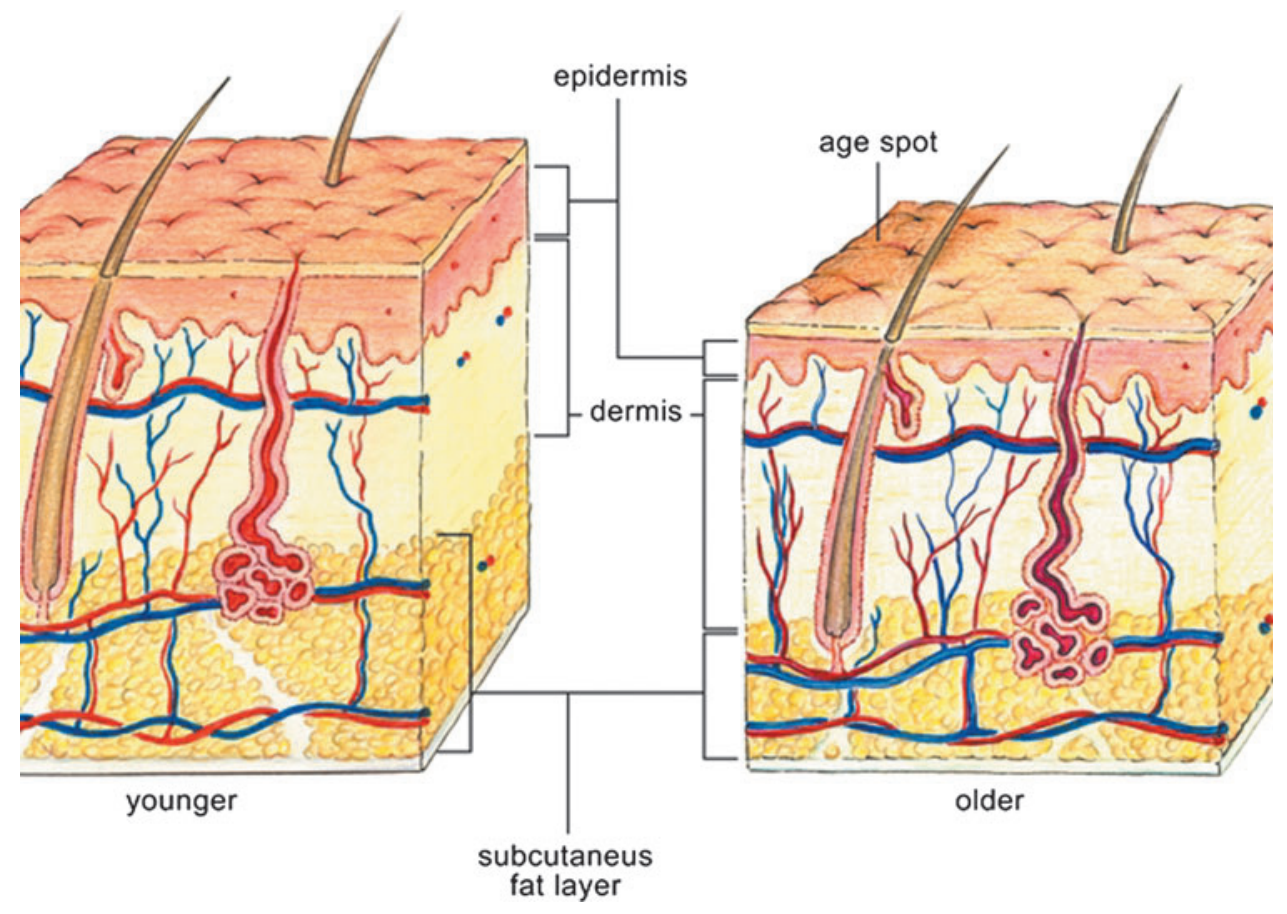

Figure 1. Differences in skin structure between younger and older skin. Color images available online at www.liebertpub.com/wound 
surface between the two layers also creates a reduced cellular supply of nutrients and oxygen, ${ }^{27}$ and an increased risk of dermo-epidermal separation, a process which may be the mechanism by which wrinkles form. ${ }^{26,27}$

Dermis thickness decreases with age $;^{15}$ thinning is accompanied by a decrease in both vascularity and cellularity. ${ }^{19}$ There is also a decrease in the number of mast cells and fibroblasts. ${ }^{12}$ The amount of glycosaminoglycans in the dermis declines with age ${ }^{26,27}$ as does the amount of hyaluronic acid produced by fibroblasts $^{26,27}$ and the amount of interfibrillary ground substance. ${ }^{29}$

Aging is inevitably associated with a decrease in collagen turnover (due to a decrease in fibroblasts and their collagen synthesis) as well as elastin. ${ }^{12}$ Elastin also has higher degree of calcification in aged skin, with an associated degradation of elastin fibers. ${ }^{17}$ Collagen crosslinks stabilize, while collagen bundles become disorganized. ${ }^{12}$

The loss of molecular integrity of the dermis leads to increased rigidity, decreased torsion extensibility, ${ }^{26}$ and diminished elasticity, ${ }^{23}$ eroding faster in women than in men, ${ }^{23}$ with a concomitant increase in vulnerability to tear-type injuries. ${ }^{26}$ Recovery from mechanical depression, in fact, is dramatically alteredobserved in only minutes in young skin, but requiring more than $24 \mathrm{~h}$ in skin of aged individuals. ${ }^{26}$

CLSM and OCT display a definite decrease in the maximal thickness of the epidermis as well as a flattening of the dermo-epidermal junction. ${ }^{26} \mathrm{~A}$ reflecting layer of fibrous structure was also observed below the basal layer, much deeper in younger than in older skin. ${ }^{26} \mathrm{~A}$ similar bright, reflecting age-associated fibrous layer in the dermis was also observed through OCT. These layers may be the transition between papillary and reticular dermis. $^{26}$

The overall volume of subcutaneous fat typically diminishes with age, although the proportion of body fat increases until approximately age 70. Fat distribution changes as well; that is, it decreases in the face, hands, and feet while a relative increase is observed in the thighs, waist, and abdomen. These changes possibly act to increase thermoregulatory function by further insulating organs. ${ }^{13}$

\section{TAKE-HOME MESSAGE}

\section{Basic science advances}

The basic mechanisms that contribute to the aging of the human integument continue to be unraveled.

- Structural stability requires stability throughout the epidermis, dermis, and hypodermis and depends on maintenance of skin thickness, collagen integrity, maintenance of a normal collagen matrix, adequate rates of skin cell turnover, maintenance of normal lipid content, and proper barrier function and hydration.

- Functional integrity of the aging skin is associated with maintenance of acidic $\mathrm{pH}$, vitamin D levels, neurosensory function, percutaneous absorption, adequate vascularization, immune surveillance, temperature regulation, and capacity for injury repair.

- Though it remains largely impenetrable to external insult, the skin changes profoundly over a lifetime, becoming progressively compromised in numerous ways with regard to both structure and function.

- As the average human lifespan continues to increase, the dermatological needs of the aging skin become more important. Utilizing the increasing knowledge of the molecular and cellular components of skin aging will facilitate more effective treatments of skin in old age.

\section{Clinical science advances}

Overall improvements in medicine, nutrition, and health awareness in industrialized countries have contributed to a steadily increasing lifespan in those areas. As the human lifespan continues to increase, the health of the human integument (the largest organ in the body) becomes more important, as do the myriad of skin disorders, which plague the elderly. Ongoing research is giving us a vastly improved understanding of the basic components of healthy skin and providing a foundation for clinical progress in the dermatological issues of the elderly.

\section{Relevance to clinical care}

Knowledge gained from basic studies of skin degeneration in old age will contribute to slow the pace of skin aging or reverse its effects. Maintaining normal cellular proliferation, reversing the intrinsic aging of cells, reversing lifestyle-related damage that contributes to premature aging, maximizing the benefits of ERT on skin while at the same time minimizing possible safety issues, and utilizing epidermal stem cell therapies are currently of interest in new therapeutical approaches.

\section{INNOVATION}

As the processes that contribute to skin aging continue to be unraveled, researchers increasingly seek to employ newly gained knowledge to develop effective antiaging therapies. Estrogen receptors are found in nearly every tissue in the body and influence the function of all organ systems in a woman. This is especially true of the largest organ, skin. Women's skin, due to the estrogen depletion associated with menopause, ages at an accelerated pace as compared with men, with both structural and functional deterioration observed. Systemic estrogen replacement therapy (ERT) has been 
shown to achieve dramatic reversals of skin aging. Estrogen therapy reverses the thinning of aging skin by both increasing collagen synthesis and retarding collagen degradation, decreases wrinkles by stimulating the synthesis of type III collagen and hyaluronic acids, and increases skin hydration and barrier function, making aging skin less dry. Despite the well-documented benefit of ERT to aging skin, concerns about the safety of long-term estrogen use to other organs have limited its usefulness. New approaches, including topical estrogens, phytoestrogens, and tissue-specific drugs called selective estrogen receptor modulators, are now being explored, and may prove valuable in maintaining optimal skin health in the postmenopausal period. ${ }^{30}$

\section{CAUTION, CRITICAL REMARKS, AND RECOMMENDATIONS}

Estrogen replacement recreates the biochemical context of youthful skin and is therefore the most promising foundation for future therapies aimed at reversing or slowing down skin aging. Women have significantly more estrogen receptors than do men and these receptors occur in virtually every female tissue. Estrogen and other sex steroids are well demonstrated to have a significant influence on skin biology and structure, with significant influence on epidermal keratinocytes, dermal fibroblasts, melanocytes, hair follicles, and sebaceous glands. Threshold levels of estrogen, in fact, are hypothesized to be critical for maintenance of skin integrity. Skin thickness has been observed to increase with rising menstrual levels of estrogen and, estrogen stimulates DNA repair. Skin aging can be significantly delayed by the administration of estrogen at menopause; moreover, nearly every structural and functional change, which accompanies the menopause in women, has been demonstrated to be at least partially reversible with ERT.

However, replacement therapy has been associated by some studies with an increased risk of cardiovascular disorders. Current research suggests that appropriate timing of replacement therapy may be key to maximizing efficacy while simultaneously minimizing associated risks. ${ }^{31}$ Extensive, well-designed clinical studies, which evaluate outcomes relative to initiation and duration of hormone replacement, are necessary for estrogen-dependent therapies targeting the physiological processes of skin aging to confidently move forward.

\section{FUTURE DEVELOPMENT OF INTEREST}

A stem cell population has been identified in the epidermis that, though smaller than originally anticipated, has the capability of reconstituting the squamous epithelium. ${ }^{32}$ This finding has obvious potential for dermatologic antiaging therapies. More researches that are recent have discovered that in fact, unlike internal stem cells, these epidermal stem cells show little evidence of normal age-dependent effects; they do not decrease in number with age, nor do they appear to suffer any loss of function. Moreover, within these epidermal stem cells, there is no evidence of changes in gene expression or developmental responsiveness or of any buildup of the ROS that contribute substantially to cellular degeneration. ${ }^{33}$ Further characterization of these cells, particularly with regard to acquiring a comprehensive of the molecular and cellular components of their unique resistance to aging, could greatly facilitate further therapies for aging skin.

\section{ACKNOWLEDGMENTS AND FUNDING SOURCES}

The authors are grateful to Drs. S. McClanahan, Randy Nunn, Keith Ertel, Don Bissett, and Joe Kaczvinsky for the critical review of this article, to Ms. Zeinab Schwen and Ms. Wendy Wippel (Strategic Regulatory Consulting, Cincinnati, OH) for writing assistance, and to Ms. Peggy Firth for the medical illustrations.

\section{AUTHOR DISCLOSURE AND GHOSTWRITING}

No conflicts of interest exist. No ghostwriters were used to write this article.

\section{REFERENCES}

1. Christensen K, Doblhammer G, Rau R, and Vaupel JW: Ageing populations: the challenges ahead Lancet 2009; 374: 1196.

2. Brincat MP, Baron YM, and Galea R: Estrogens and the skin. Climacteric 2005; 8: 110.
3. Velarde MC, Flynn JM, Day NU, Melov S, and Campisi J: Mitochondrial oxidative stress caused by Sod2 deficiency promotes cellular senescence and aging phenotypes in the skin. Aging 2012; 4: 3.

4. Glogau RG: Systemic evaluation of the aging face. In: Dermatology, edited by Bolognia JL, Jorizzo JL, and Rapini RP. Edinburgh: Mosby, 2003, pp. 2357-2360

5. Menon $G$ and Ghadially R: Morphology of lipid alterations in the epidermis: a review. Microsc Res Tech 1997; 37: 180. 
6. Vierkötter A, Schikowski T, Ranft U, Sugiri D, Matsui $\mathrm{M}$, Krämer $\mathrm{U}$, and Krutmann J: Airborne particle exposure and extrinsic skin aging. J Invest Dermatol 2010; 130: 2719.

7. Kligman AM and Koblenzer C: Demographics and psychological implications for the aging population. Dermatol Clin 1997; 15: 549.

8. Farage MA, Miller KW, Berardesca E, and Maibach HI: Clinical implications of aging skin: cutaneous disorders in the elderly. Am J Clin Dermatol 2009; 10: 73.

9. Farage MA, Miller KW, Berardesca E, and Maibach $\mathrm{HI}$ : Neoplastic skin lesions in the elderly patient. Cutan Ocul Toxicol 2008; 27: 213.

10. Farage MA, Miller KW, Elsner P, and Maibach $\mathrm{HI}$ : Functional and physiological characteristics of the aging skin. Aging Clin Exp Res 2008; 20: 195 (Review).

11. Krueger N, Luebberding S, Oltmer M, Streker M, and Kerscher $\mathrm{M}$ : Age-related changes in skin mechanical properties: a quantitative evaluation of 120 female subjects. Skin Res Technol 2011; 17: 141.

12. Duncan KO and Leffell DJ: Preoperative assessment of the elderly patient. Dermatol Clin 1997; 15: 583.

13. Farage MA, Miller KW, and Maibach HI: Degenerative changes in aging skin. In: Textbook of Aging Skin, edited by Farage MA, Miller KW, and Maibach HI. Berlin/Heidelberg: Springer-Verlag, 2010, pp. 25-35.

14. Grove GL: Physiologic changes in older skin. Clin Geriatr Med 1989; 5: 115

15. Waller JM and Maibach HI: Age and skin structure and function, a quantitative approach (I): blood flow, pH, thickness, and ultrasound echogenicity. Skin Res Technol 2005; 11: 221.
16. Harvell JD and Maibach HI: Percutaneous absorption and inflammation in aged skin: a review. J Am Acad Dermatol 1994; 31: 1015.

17. Boss GR and Seegmiller JE: Age-related physiological changes and their clinical significance. West J Med 1981; 135: 434.

18. Oriba HA, Bucks DA, and Maibach HI: Percutaneous absorption of hydrocortisone and testosterone on the vulva and forearm: effect of the menopause and site. Br J Dermatol 1996; 134: 229.

19. Suter-Widmer $J$ and Elsner P: Age and irritation. In: The Irritant Contact Dermatitis Syndrome, edited by Agner T and Maibah H. Boca Raton, FL: CRC Press, 1996, pp. 257-265.

20. Phillips $T$ and Kanj L: Clinical manisfestations of skin aging. In: The Effect of Aging in Oral Mucosa and Skin, edited by Squier $C$ and Hill MW. Boca Raton, FL: CRC Press, 1994, pp. 25-40.

21. Fiers SA: Breaking the cycle: the etiology of incontinence dermatitis and evaluating and using skin care products. Ostomy Wound Manag 1996; 42: 32.

22. Jackson SM, Williams ML, Feingold KR, and Elias PM: Pathobiology of the stratum corneum. West $J$ Med 1993; 158: 279.

23. McCallion R and Li Wan Po A: Dry and photo-aged skin: manifestations and management. J Clin Pharm Ther 1993; 18: 15.

24. Ghadially R: Aging and the epidermal permeability barrier: implications for contact dermatitis. Am J Contact Dermat 1998; 9: 162.

25. Ghadially R, Brown BE, Sequeira-Martin SM, Feingold KR, and Elias PM: The aged epidermal permeability barrier. Structural, functional, and lipid biochemical abnormalities in humans and a senescent murine model. J Clin Invest 1995; 95: 2281.

26. Martini F: Fundamentals of Anatomy and Physiology. San Francisco: Benjamin-Cummings, 2004.
27. Südel KM, Venzke $K$, Mielke $H$, Breitenbach $U$ Mundt C, Jaspers S, Koop U, Sauermann K, Knussman-Hartig E, Moll I, Gercken G, Young AR, Stäb F, Wenck H, and Gallinat S: Novel aspects of intrinsic and extrinsic aging of human skin: beneficial effects of soy extract. Photochem Photobiol 2005; 81: 581.

28. Neerken S, Lucassen GW, Bisschop MA, Lenderink $E$, and Nuijs TAM: Characterization of agerelated effects in human skin: a comparative study that applies confocal laser scanning microscopy and optical coherence tomography. J Biomed Opt 2004; 9: 274

29. Castelo-Branco C, Figueras F, Martínez de Osaba MJ, and Vanrell JA: Facial wrinkling in postmenopausal women. Effects of smoking status and hormone replacement therapy. Maturitas 1998; 29: 75

30. Shu YY and Maibach HI: Estrogen and skin: therapeutic options. Am J Clin Dermatol 2011; 12: 297

31. Rosano GMC, Maffei S, Andreassi MG, Vitale $C$, Vassalle C, Gambacciani M, Stramba-Badiale M, and Mercuro G: Hormone replacement therapy and cardioprotection: a new dawn? A statement of the Study Group on Cardiovascular Disease in Women of the Italian Society of Cardiology on hormone replacement therapy in postmenopausal women. J Cardiovasc Med 2009; 10: 85 .

32. Schneider TE, Barland C, Alex AM, Mancianti ML, Lu Y, Cleaver JE, Lawrence HJ, and Ghadially R: Measuring stem cell frequency in epidermis: a quantitative in vivo functional assay for long-term repopulating cells. Proc Natl Acad Sci USA 2003; 100: 11412.

33. Racila D and Bickenbach JR: Are epidermal stem cells unique with respect to aging? Aging 2009; 1: 746 . 